Original Research Article

\title{
A clinical study to determine metformin as a cause of serum vitamin B12 decrease and effect of combination of metformin and mecobalamin on serum vitamin B12 levels in type 2 diabetics
}

\author{
Mayuresh D. Kiran ${ }^{1 *}$, Bhagyashree N. Naik ${ }^{2}$
}

\begin{abstract}
${ }^{1}$ Senior Pharmacologist and General Manager, ${ }^{2}$ Officer, Medical Services, Centaur Pharmaceuticals Private Limited, Mumbai, Maharashtra, India
\end{abstract}

Received: 08 March 2017

Accepted: 22 March 2017

*Correspondence to:

Dr. Mayuresh D. Kiran,

Email:

mayuresh_kiran@rediffmail.co

$\mathrm{m}$

Copyright: () the author(s), publisher and licensee Medip Academy. This is an openaccess article distributed under the terms of the Creative Commons Attribution NonCommercial License, which permits unrestricted noncommercial use, distribution, and reproduction in any medium, provided the original work is properly cited.

\begin{abstract}
Background: Several evidences show metformin decreases vitamin B12. Diabetics are also at the risk of developing neuropathy which is treated with vitamin B12. metformin being initial therapy for diabetic patients, it becomes all the more important to know the extent of this decrease. The study was designed with the objective to compare the levels of vitamin B12 in patients on metformin vis-à-vis on antidiabetic drugs other than metformin and to evaluate the increase in vitamin B12 levels after prescribing a combination of metformin and mecobalamin, in T2DM patients.

Methods: Of 500 enrolled, 321 patients completed the study for duration of 6 months, divided in two periods of 3 months each. At the end first period of 3 months the vitamin B12 levels were compared form Metformin vs. that of other antidiabetics. In second period of 3 months a combination of metformin and mecobalamin was given instead of plain metformin and vitamin B12 levels were repeated at the end of this period.

Results: There was reduction in vitamin B12 levels with metformin with levels of $272.5 \mathrm{pg} / \mathrm{ml}$ compared to $714.6 \mathrm{pg} / \mathrm{ml}$ with other antidiabetics at the end of first period. The levels increased from $272.5 \mathrm{pg} / \mathrm{ml}$ to $615.9 \mathrm{pg} / \mathrm{ml}$ at the end of second period after receiving the combination of metformin and mecobalamin.

Conclusions: T2DM patients being treated with metformin had a greater risk of reduction in vitamin B12 levels and addition of vitamin B12 in the form of mecobalamin would decrease the reduction in vitamin B12 levels associated with Metformin therapy.
\end{abstract}

Keywords: Diabetes, Metformin, Mecobalamin, Vitamin B12

\section{INTRODUCTION}

Prevalence of Type 2 Diabetes Mellitus (T2DM) is increasing all over the globe at an alarming rate. A recent survey of International Diabetes Federation reported, around 415 million of people with DM in 2015 and further projected to rise to about 642 million by $2040 .{ }^{1}$ It is also the most common endocrine disorder accounting for 69.1 million people with T2DM cases in India, making India the diabetes capital of the world. ${ }^{1}$ T2DM involves several factors playing a pioneer role in its progression most likely, sedentary lifestyle, dearth of physical exercise, increased life expectancy, obesity and earlier diagnosis to a certain extent. ${ }^{2}$ T2DM is driven by series of etiologies associated with chronic hyperglycemia and insulin resistance. ${ }^{3}$

Insulin resistance is the reduced responsiveness of a target cell to the insulin concentration to which it is exposed. ${ }^{4}$ Important factor associated with an increased risk of developing insulin resistance in T2DM is obesity. ${ }^{5}$ Thus, insulin resistance and obesity are the driving force in the progression of diabetes, requiring importance in the therapy in T2DM.

American Diabetes Association and the European Association for the Study of Diabetes have recommended, Metformin as initial medical therapy for T2DM. ${ }^{6}$ Unlike 
the other anti-hyperglycaemic agents, metformin is reported to show improvements in cardiovascular $(\mathrm{CV})$ morbidity and mortality, which is a one of the major cause of death in patients with T2DM. ${ }^{7,8}$ It is known that metformin demonstrates an effect of hepatic gluconeogenesis suppression. ${ }^{9}$ Further, reduction in serum glucose is also a consequence of its action on several tissues via activation of the adenosine monophosphateactivated protein kinase (AMPK) system, reducing insulin resistance. ${ }^{9}$ Additionally, metformin has beneficial effects on carbohydrate metabolism, weight loss, and vascular protection. ${ }^{10}$ Despite its known efficacy and satisfactory safety profile, it has non-negligible side effect such as decreased serum vitamin B12 levels. The magnitude of decrease in serum vitamin B12 concentration associated with metformin therapy, is directly proportional to the dose of metformin used. ${ }^{11}$ The prevalence of B12 deficiency varies from $5.8 \%$ to $30 \%$ among patients undergoing long-term treatment with Metformin. ${ }^{12,13}$

The actual mechanism behind vitamin B12 deficiency associated with metformin therapy is yet not clear. There are certain postulations demonstrating that vitamin B12 deficiency is due to either alteration in small bowel motility, which stimulate small bowel bacterial overgrowth and/or by decreasing vitamin B12 absorption. ${ }^{14,15}$ Drop in vitamin B12 concentrations in turn, might results in rise in homocysteine (Hcy) concentrations, an independent risk factor for cardiovascular disease, particularly among individuals with T2DM. ${ }^{16}$

Vitamin B12 is one of the vital nutrients required for the proper functioning of the nervous system, cardiovascular system and hemopoesis. ${ }^{17,18}$ Apart from causing megaloblastic anemia, vitamin B12 deficiency may also increase the severity of peripheral neuropathy in patients with T2DM. Diabetic peripheral neuropathy (DPN) is the common complication seen with hyperglycemic patient, with annual incidence rate $\sim 2 .{ }^{19}$ The pathology of diabetic neuropathy encompasses series of stress factors likely, oxidative stress, advanced glycation end products, polyol pathway flux and protein kinase $\mathrm{C}$ activation. ${ }^{20}$ Moreover, vitamin B12 decrease due to metformin would further aggravate neuropathy which is already seen in the diabetic patient.

mecobalamin is the neurologically active form of vitamin B12. ${ }^{21}$ It is claimed to relieve clinical symptoms and signs of DPN thus, improving the nerve conduction velocity and quality of life. ${ }^{22}$ Interestingly, this methyl-base-attached cobalamin (methycobalamin) has a special affinity for nerve tissues to promote myelination and transport of axonal cytoskeleton which has significance in nerve repair. $^{23}$

The current study was designed with an objective to compare the levels of vitamin B12 in patients on metformin vis-à-vis on antidiabetic drugs other than metformin and to evaluate the increase in vitamin B12 levels after prescribing a combination of metformin and mecobalamin, in T2DM patients.

\section{METHODS}

A systematic, multi-centered, comparative clinical study was planned with the abovementioned dual objective. This study was conducted at 21 centers across India. A total of 500 T2DM patients were recruited for the study. This study was conducted from October 2015 to November 2016.

\section{Inclusion and exclusion criteria}

This study included patients of both the gender having age between age $\geq 18$ years. Patients with confirmed diagnosis of T2DM and were previously on Metformin or any other antidiabetic therapy were included in the study. Only the patients who would strictly adhere to the protocol were recruited for the study. Type I Diabetes Mellitus patients were generally excluded from the study. Patients with hypersensitivity to the individual study drugs, patients having hepatic and renal impairment were also excluded. This study also excluded pregnant, lactating women and patients with any evidence of psychological disorder.

\section{Study drug}

A tablet containing a combination of metformin $500 \mathrm{mg}+$ mecobalamin $750 \mathrm{mcg}$ was provided by the sponsor free of cost to the patient. Study drug was administered as one tablet which was taken 2 times in a day at 12 hourly intervals during meals with a glass of water.

\section{Study procedure}

The study duration was decided to be 6 months which was divided into two periods of 3 months each. Patients satisfying the inclusion and exclusion criteria were recruited for the study. A detailed medical history was taken and physical examination (including the vital signs, systemic and general examination) was conducted by the investigators. In the first period of 3 months' patient continued to take their medicines as prescribed by the physician either metformin or antidiabetic drugs other than metformin. Serum vitamin B12 levels were done after this period of 3 months. The study concluded for patients who were on antidiabetic drugs other than metformin. For the patients who were on metformin the second period of 3 months began. These patients were dispensed 180 tablets of the study drug (3 months therapy) by the investigator. Patients were asked to maintain a symptoms diary and note any adverse events occurring during the entire study duration. Three visits were planned for the patients recruited in this study-V1 (0-month visit), V2 (3-month visit) and V3 (6 month visit only for the patients in the Metformin group). Change in vitamin B12 levels and adverse events occurring were noted during each visit along with medical history and clinical examination. 
Investigators were asked to discontinue the study drug in case of severe adverse event and with discretion in case of mild to moderate adverse events (Table 1).

Table 1: Visits by both the groups (group A and group B).

\begin{tabular}{|lll|}
\hline Visit 1 (0 month) & Group A (other antidiabetic drugs group) & Group B (metformin group) \\
\hline Visit 2 (3 months) & Vitamin B12 levels; end of study & $\begin{array}{l}\text { Vedical history and clinical examination } \\
\text { of Metformin and mecobalamin }\end{array}$ \\
\hline Visit 3 (6 months) & - & Vitamin B12 levels; end of study \\
\hline
\end{tabular}

\section{Efficacy assessment}

Efficacy assessment was done by measuring the vitamin B12 level at the study visits 2 and 3 (only for group B). Primary and secondary parameters were evaluated. Primary parameters included-(a) effect of Metformin and other anti-diabetic therapy on serum vitamin B12 levels and (b) effect of the combination of metformin and mecobalamin on serum vitamin B12 levels. Secondary parameters included-(a) percentage of patients having vitamin B12 deficiency $(<200 \mathrm{pg} / \mathrm{ml})$ with metformin monotherapy and (b) percentage of patients with double serum vitamin B12 levels after switch of Metformin and mecobalamin combination in 3 months.

\section{Safety assessment}

Adverse events occurring during the clinical trial were noted in the case record forms and analyzed.

\section{Concomitant therapy}

Pharmacological intervention and medication including other antidiabetics, anti-cholesterol and anti-hypertensives were allowed during the study duration. No other multivitamin and multi-mineral was allowed during the study interval. Non-Pharmacological interventions like exercise and proper diabetic diet intake at regular intervals were allowed and encouraged during the study duration.

All the patients involved in this clinical study signed an informed consent form. The protocol of the study was approved by the institutional ethics review board and with the 1964 Helsinki declaration and its later amendments or comparable ethical standards.

\section{Statistical analysis}

All the patients who completed the study were included in the efficacy analysis set. The data was recorded and analyzed using statistical test comparisons were performed using analysis of covariance (ANCOVA).

\section{RESULTS}

A total of 500 patients were recruited at 21 centres across India, 321 patients completed the study and were analyzed. 179 patients were lost to follow up (Figure 1).

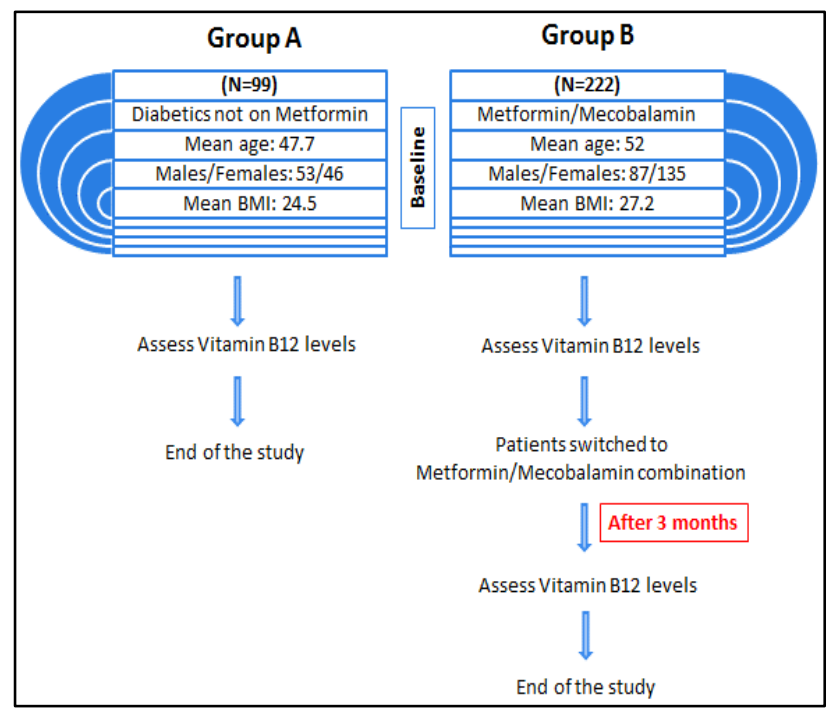

Figure 1: Schematic representation of study design (consort flow chart).

\section{Efficacy assessment}

\section{Primary parameters}

- Effect of Metformin and other anti-diabetic therapy on serum vitamin B12 levels (first period)

The mean serum B12 concentration was $714.9 \mathrm{pg} / \mathrm{ml}(\mathrm{P}<$ $0.05)$ in those with T2DM on other antidiabetic drugs not on Metformin as compared to $272.5 \mathrm{pg} / \mathrm{ml}$ in the Metformin group (Figure 2), after the end of first period of 3 months. This difference was statistically significant ( $p$ $<0.05)$.

- Effect of metformin and mecobalamin combination therapy on serum vitamin B12 levels (second period) 
Patients in Group B (metformin Group) were given the combination of metformin and mecobalamin for second period of 3 months and the serum vitamin B12 levels were increased to $615.9 \mathrm{pg} / \mathrm{ml}$ from the level of $272.5 \mathrm{pg} / \mathrm{ml}$ (Figure 3 ) in the first period of 3 months. This difference was statistically significant ( $\mathrm{p}<0.05)$.

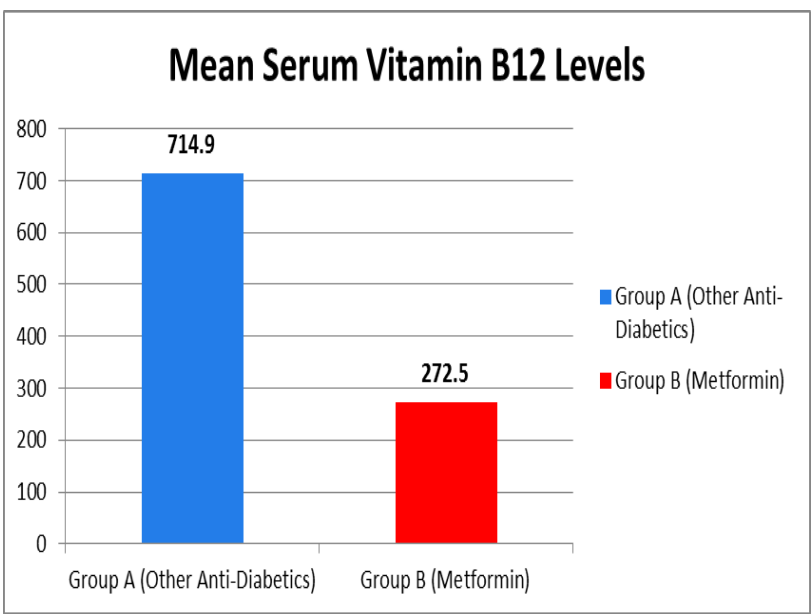

Figure 2: Effect of metformin and other anti-diabetic therapy on vitamin B12 levels (first period).

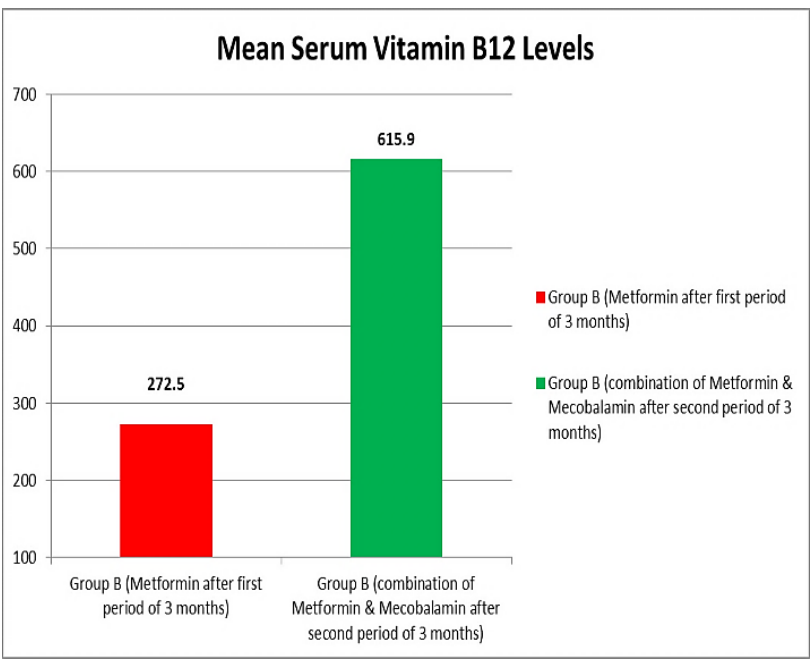

Figure 3: Effect of metformin and mecobalamin combination on vitamin B12 levels (second period).

\section{Secondary parameters}

A study conducted by Carmel et al, defined biochemical B12 deficiency as serum levels $\leq 148 \mathrm{pmol} / \mathrm{L}$, borderline deficiency as serum B12>148 to $\leq 221 \mathrm{pmol} / \mathrm{L}$, and normal as $>221 \mathrm{pmol} / \mathrm{L}^{24}$ Hence, the variation in vitamin B12 levels were assessed during and after the metformin therapy (alone and combination).

(a) Percentage of patients having vitamin B12 deficiency ( $<200 \mathrm{pg} / \mathrm{ml})$ with Metformin monotherapy
In 71 patients had low vitamin B12 levels $<200 \mathrm{pg} / \mathrm{ml}$ at baseline with metformin monotherapy which accounted for $32 \%$ of patients. None of the patients on antidiabetics other than metformin had vitamin B12 deficiency.

(b) Percentage of patients with double serum vitamin $B 12$ levels after switch of metformin and mecobalamin combination in 3 months

In 137 patient's vitamin B12 levels was doubled after 3 months of metformin and mecobalamin combination which accounted for $62 \%$ of patients. This indicated the combination was effective in ameliorating the low levels of vitamin B12 associated with plain Metformin therapy. None of the patients who earlier had vitamin B12 deficiency when on plain Metformin had vitamin B12 deficiency after switching to the combination of metformin and mecobalamin.

\section{Safety assessment}

There were no serious adverse events reported during the study. The adverse events of this study are reported in Table 2.

Table 2: Adverse events occurred during study.

\begin{tabular}{|ll|}
\hline Adverse events & Incidences (\%) \\
\hline Flatulence & $22(10 \%)$ \\
\hline Gastritis and heart burn & $20(9 \%)$ \\
\hline Diarrhoea & $15(7 \%)$ \\
\hline Constipation & $6(3 \%)$ \\
\hline Anorexia & $4(2 \%)$ \\
\hline Hypoglycemia & $2(1 \%)$ \\
\hline
\end{tabular}

None of the patients had to discontinue the study medication due to the reported adverse events.

\section{DISCUSSION}

This multi-centered comparative study conducted in T2DM patients, to the authors knowledge this was the first comparative clinical trial to study the effects of metformin and other antidiabetic drugs on serum vitamin B12 in Indian patients. Indian patients being vegetarian or mostly vegetarian have more tendencies to develop vitamin B12 deficiency. ${ }^{25}$

Moreover, there are studies stating metformin in short and long-term therapy is associated with deficiency of vitamin B12. ${ }^{13,26}$ Thus it becomes important to know the effect of metformin in on serum vitamin B12 levels in Indian patients. Further, these are no studies comparing the effects of combination of metformin and mecobalamin on serum vitamin B12 levels with that of plain metformin therapy in T2DM patients, in the author's knowledge. This study was two pronged, first of its kind conducted in 321 Indian T2DM patients. 
This study documents metformin as a cause for decreased levels of vitamin B12 as the metformin group in the first period of 3 months had significantly lower levels of serum vitamin B12 as compared to the group with antidiabetic drugs other than metformin. Nearly $1 / 3$ rd of the patients in the metformin group had deficiency of vitamin B12 (levels less than $200 \mathrm{pg} / \mathrm{ml}$ ). Upon changing metformin to a combination of metformin and mecobalamin during second period of the study the levels of serum vitamin B12 increased significantly and in nearly $2 / 3$ rd patients the levels doubled.

Bauman WA et al, in a 21-patient study have proved the decrease of serum vitamin B12 by $29.5 \%$ (p <0.0005) in just 3 months, which is a short duration of therapy with metformin. ${ }^{27}$ Most of the patients of T2DM have to take antidiabetic drugs including Metformin for lifetime. Wulffele et al, conducted a 16-week study on 745 T2DM patients resulting in decrease of serum vitamin B12 levels from $390.1 \mathrm{pg} / \mathrm{ml}$ to $332.6 \mathrm{pg} / \mathrm{ml}$ with an additional increase on homocysteine of $4 \% .^{28}$

Similarly, in long-term studies, Jagger $\mathbf{J}$ et al in a 390 T2DM placebo controlled trial for 4.3 years, showed Metformin had higher chances of vitamin B12 deficiency and raised homocysteine levels. ${ }^{2}$ Hermann et al, in another long-term study for 12 months showed significantly $(p<0.01)$ lower levels of serum vitamin B12 levels $(289 \mathrm{pg} / \mathrm{ml})$ as compared to other antidiabetic drugs $(395 \mathrm{pg} / \mathrm{ml}){ }^{29}$

Damião $\mathrm{CP}$ et al, conducted a cross sectional clinical trial with 462 T2DM patients on metformin in Brazil. ${ }^{30}$ The study demonstrated vitamin B12 deficiency in $22.5 \%$ of patients on metformin as compared to $7.4 \%$ of controls. The mean serum vitamin B12 was $272 \mathrm{pg} / \mathrm{ml}$ and was significantly ( $\mathrm{p}<0.001$ ) lower than controls with 348 $\mathrm{pg} / \mathrm{ml}$. In another cross-sectional study by Qureshi et al., documented a high prevalence of vitamin B12 deficiency of $33 \%$ among adult patients with T2DM. However, patients enrolled in this study were those who were on high dose (>2 g/day) and long-term (4 years) metformin treatment, both clinical factors known to be associated with vitamin B12 deficiency. ${ }^{31}$

Additionally, Wile DJ et al, in a prospective case-control study conducted with 122 T2DM patients demonstrated Metformin treated patients had depressed cobalamin levels (231 pmol/L vs. 436 pmol/L for controls; p <0.001) and elevated fasting methylmalonic acid (MMA) and homocysteine levels. ${ }^{26}$ Cobalamine deficiency was seen in $31 \%$ of patients on Metformin as opposed to $3 \%$ in case of controls $(\mathrm{p}<0.001)$. this decrease and deficiency of cobalamin further translates to neuropathy with NIS score of 10 in metformin group and 4 in the control group ( $p$ $<0.001)$.

Vitamin B12 or mecobalamin treatment would possibly correct the deficiency state and reverse the related neuropathy, caused due to Metformin. A meta-analysis involving 1949 patients indicated that mecobalamin showed significantly positive effects on the improvement of the signs and symptoms of DPN and the effects were better than the other vitamin $\mathrm{B}$ agents. Also, the increase of nerves conduction velocities by mecobalamin was better than by the other vitamin B supplements. ${ }^{22}$ The study also demonstrated that deficiency of vitamin B12 with metformin as first-line therapy drug can progress to series of etiologies and thus, supplement with vitamin B12 can be rational.

Thus, present study may prove that the combination of metformin and mecobalamin has a role in the treatment of T2DM, to attenuate the vitamin B12 decrease caused by plain Metformin. Also, the combination would increase the vitamin B12 levels in the body leading to betterment in the symptoms of DPN.

\section{Limitations}

Limitations of our study include the fact that we measured only total serum vitamin B12 levels and not levels of holotranscobalamin II or methylmalonic acid or homocysteine. Another limitation of our study would be that we did not test clinical neuropathy and betterment in neuropathy symptoms, but this was beyond the scope of our study.

\section{CONCLUSION}

In conclusion, we showed that in patients with T2DM being treated with Metformin alone had a greater risk of reduction in vitamin B12 levels. Moreover, addition of vitamin B12 in the form of mecobalamin would decrease the reduction in vitamin B12 levels associated with metformin therapy. Current guidelines throw light on metformin, a cornerstone in the treatment of T2DM, but make no recommendations on the detection and prevention of vitamin B12 decrease during metformin treatment. The findings of this current study provided a strong case for predictable assessment of vitamin B12 levels during treatment with Metformin.

\section{ACKNOWLEDGEMENTS}

This study was drafted with the medical writing help received from Medical Services Department of Centaur Pharmaceuticals Pvt Ltd.

\section{Funding: Centaur Pharmaceuticals Pvt. Ltd Conflict of interest: None declared \\ Ethical approval: The study was approved by the Institutional Ethics Committee}

\section{REFERENCES}

1. International Diabetes Federation. IDF Diabetic Atlas. $7^{\text {th }}$ Ed. Brussels, Belgium: International Diabetes Federation; 2015. 
2. de Jager J, Kooy A, Lehert P, Wulffelé MG, van der Kolk J, Bets D et al. Long term treatment with Metformin in patients with type 2 diabetes and risk of vitamin B-12 deficiency: randomised placebo controlled trial. BMJ. 2010;340:c2181.

3. Pandey AK, Pandey D, and Pandit A. Obesity and Lipid Profile Study in Type 2 Diabetes Patients with Auditory and Reaction Time Deficits and Nondiabetic Control Subjects. Adv Diab Metab. 2017:5;1:1-5.

4. Shanik MH, Xu Y, Škrha J, Dankner R, Zick Y, Roth J. Insulin resistance and hyperinsulinemia. Diabetes Care. 2008;31(2):262-8.

5. Kahn SE, Hull RL, Utzschneider KM. Mechanisms linking obesity to insulin resistance and type 2 diabetes. Nature. 2006;444(7121):840-6.

6. Nathan DM, Buse JB, Davidson MB, Heine RJ, Holman RR, Sherwin R, et al. Management of hyperglycaemia in type 2 diabetes: a consensus algorithm for the initiation and adjustment of therapy. Diabetologia. 2006;49(8):1711-21.

7. Hermansen $\mathrm{K}$, Mortensen LS, Hermansen $\mathrm{M}$. Combining insulins with oral antidiabetic agents: effect on hyperglycemic control, markers of cardiovascular risk and disease. Vascular Health and Risk Management. 2008;4(3):561.

8. Kooy A, de Jager J, Lehert P, Bets D, Wulffele MG, Donker AJM, et al. Long-term effects of Metformin on metabolism and microvascular and macrovascular disease in patients with type 2 diabetes mellitus. Arch Intern Med. 2009;169:616-25.

9. Diamanti-Kandarakis E, Christakou CD, Kandaraki E, Economou FN. Metformin: an old medication of new fashion: evolving new molecular mechanisms and clinical implications in polycystic ovary syndrome. Eur J Endocrinol. 2009;162(2):193-212.

10. UK Prospective Diabetes Study (UKPDS) Group. Effect of intensive blood-glucose control with Metformin on complications in overweight patients with type 2 diabetes (UKPDS 34).1998;352:854-65.

11. Ting RZ, Szeto CC, Chan MH, Ma KK, Chow KM Risk factors of vitamin B12 deficiency in patients receiving Metformin. Arch Intern Med. 2006;166:1975-9.

12. Pflipsen MC, Oh RC, Saguil A. The prevalence of vitamin B (12) deficiency in patients with type 2 diabetes: a cross-sectional study. J Am Board Fam Med. 2009;22(5):528-34.

13. Reinstatler L, Qi YP, Williamson RS, Garn JV, Oakley GP. Association of biochemical B12 deficiency with Metformin therapy and vitamin B12 supplements: the National Health and Nutrition Examination Survey, Diabetes Care. 2012;35(2):32733.

14. Diamanti-Kandarakis E, Christakou CD, Kandaraki E, Economou FN. Metformin: an old medication of new fashion: evolving new molecular mechanisms and clinical implications in polycystic ovary syndrome. Eur J Endocrinol. 2009;162(2):193-212.
15. Liu KW, Dai LK, Jean W. Metformin-related vitamin B12 deficiency. Age and Ageing. 2006;35(2):200-1.

16. Hoogeveen EK, Kostense PJ, Beks PJ, Mackaay AJ, Jakobs C, Bouter LM et al. Hyperhomocysteinemia is associated with an increased risk of cardiovascular disease, especially in non-insulin-dependent diabetes mellitus. Arterioscler Thrombo Vascular Biol. 1998;18(1):133-8.

17. Liu Q, Li S, Quan H, Li J. Vitamin B 12 status in Metformin treated patients: systematic review. PloS one. 2014;9(6):100379.

18. Kibirige D, Mwebaze R. Vitamin B12 deficiency among patients with diabetes mellitus: is routine screening and supplementation justified? J Diab Metab Disord. 2013;12(1):17.

19. Duby JJ, Campbell RK, Setter SM, Rasmussen KA. Diabetic neuropathy: an intensive review. Am J Health-Syst Pharmacy. 2004;61(2):160-73.

20. Giacco F, Brownlee M. Oxidative stress and diabetic complications. Circulation research. 2010;107(9):1058-70.

21. Ide H, Fujiya S, Asanuma Y, Tsuji M, Sakai H, Agishi Y. Clinical usefulness of intrathecal injection of methylcobalamin in patients with diabetic neuropathy. Clinical therapeutics. 1986;9(2):183-92.

22. Hairyan JIA., Haorming and Dong. Effects of methylcobalamin on diabetic peripheral neuropathy: a systematic review. Chinese J Evidence Based Medicine. 2005;5(8).

23. Mizukami H, Ogasawara S, Yamagishi SI, Takahashi $\mathrm{K}$, Yagihashi S. Methylcobalamin effects on diabetic neuropathy and nerve protein kinase $\mathrm{C}$ in rats. European J Clini Investi. 2011;41(4):442-50.

24. Carmel R, Green R, Rosenblatt DS, Watkins D. Update on cobalamin, folate, and homocysteine. ASH Education Program Book. 2003;2003(1):62-81.

25. Herrmann W, Schorr H, Obeid R, Geisel J. Vitamin B12 status, particularly holotranscobalamin II and methylmalonic acid concentrations, and hyperhomocysteinemia in vegetarians. Am J Clin Nutr. 2003;78(1):131-6.

26. Wile DJ, Toth C. Association of Metformin, elevated homocysteine, and methylmalonic acid levels and clinically worsened diabetic peripheral neuropathy. Diabetes Care. 2010;33(1):156-61.

27. Bauman WA, Shaw S, Jayatilleke E, Spungen AM, Herbert V. Increased intake of calcium reverses vitamin B12 malabsorption induced by Metformin. Diabetes Care. 2000;23:1227-31.

28. Wulffele MG. Effects of short-term treatment with Metformin on serum concentrations of homocysteine, folate and vitamin B12 in type 2 diabetes mellitus: a randomized, placebo-controlled trial. $\mathrm{J}$ Internal Medicine. 2003;254:455-63.

29. Hermann LS. Vitamin B12 status of patients treated with Metformin: a cross-sectional cohort study, The Br J Diab Vascu Disease. 2004;4(6).

30. Damião CP, Rodrigues AO, Pinheiro MF, Cruz Filho RA, Cardoso GP, et al. Prevalence of vitamin B12 deficiency in type 2 diabetic patients using Metformin: 
a cross-sectional study. Sao Paulo Medic J. 2016;134(6):473-9.

31. Qureshi SA, Ainsworth A, Winocour PH. Metformin therapy and assessment for vitamin B12 deficiency: is it necessary? Prac Diab. 2011;28(7):302-4.
Cite this article as: Kiran MD, Naik BN. A clinical study to determine metformin as a cause of serum vitamin B12 decrease and effect of combination of metformin and mecobalamin on serum vitamin B12 levels in type 2 diabetics. Int $\mathrm{J}$ Basic Clin Pharmacol 2017;6:1066-72. 